\title{
Solar magnetic field reversal as seen at Ulysses
}

\author{
Geraint H. Jones ${ }^{1}$ and André Balogh \\ Space and Atmospheric Physics Group, The Blackett Laboratory, Imperial College, London, UK
}

Edward J. Smith

Jet Propulsion Laboratory, Pasadena, California, USA

Received 25 February 2003; revised 14 May 2003; accepted 5 June 2003; published 11 September 2003.

[1] The rapid motion of the Ulysses spacecraft from high southern to high northern latitudes in 2000-2001 provided an excellent opportunity to make inferences regarding the solar magnetic dipole's behaviour around solar maximum. A simple dipole model is fitted to Ulysses measurements of the polarity of the heliospheric magnetic field mapped back to the solar wind source surface. Although higher order components of the field are ignored, the gradual reversal in orientation of the dipole field component can be followed during solar maximum, with the dipole axis crossing the solar equator during early $2000-$ early 2001 . The dipole appears to exhibit a rotation at a slower rate than the Carrington frame of reference, similar to previous measurements made around solar maximum in the solar equatorial regions. INDEX TERMS: 2134 Interplanetary Physics: Interplanetary magnetic fields; 2162 Interplanetary Physics: Solar cycle variations (7536); 7524 Solar Physics, Astrophysics, and Astronomy: Magnetic fields; 7536 Solar Physics, Astrophysics, and Astronomy: Solar activity cycle (2162). Citation: Jones, G. H., A. Balogh, and E. J. Smith, Solar magnetic field reversal as seen at Ulysses, Geophys. Res. Lett., 30(19), 8028, doi:10.1029/2003GL017204, 2003.

\section{Introduction}

[2] The period of maximum solar activity has long been recognized as the time when the Sun's large-scale magnetic field reverses polarity. As most spacecraft's orbits are nearecliptic, in-situ observations of the Sun's open magnetic flux, carried outwards with the solar wind as the heliospheric magnetic field (HMF), have mostly been obtained at low heliolatitudes. A global view of the open magnetic flux's polarity distribution has thus relied on models of the solar wind source surface, at $\sim 2.5$ solar radii, based upon observations of the photospheric field. Good agreement has been found between these models and low-latitude HMF observations, but at high latitudes, these models are limited in accuracy, due to difficulties in observing the underlying high-latitude photospheric fields.

[3] The ESA/NASA Ulysses spacecraft, in its high-inclination orbit, detects the HMF over a wide heliolatitude range $\left( \pm 80.1^{\circ}\right)$. Around perihelion, it performs a pole to pole scan in only 10.5 months. The first of these was near solar minimum, but the latest coincided with solar maximum, recording in-situ the global distribution of open magnetic flux while the solar magnetic field reversal took

\footnotetext{
${ }^{1}$ Now at Jet Propulsion Laboratory, Pasadena, CA.
}

place. Here, Ulysses data are used to deduce the behaviour of the dipolar component of the solar magnetic field around solar maximum, showing the reversal to be largely consistent with a simple rotation of the dipole field component. The observations also show that the magnetic dipole field during this period was rotating at a slower rate than the Carrington reference frame.

[4] An overview of the HMF polarity distribution at Ulysses from early 1992 to late 2002 is given by Jones and Balogh [2003]. The polarities during the analysis period are shown in Figure 1. Following Ulysses's first north polar pass, almost unipolar fields were detected until a heliolatitude of $25.5^{\circ} \mathrm{N}$ [Forsyth et al., 1997]. South of the equator, Ulysses continued to detect both polarities, with a four-sector structure appearing at the beginning of this period. A sudden change appeared in the distribution when Ulysses was at $\sim 30^{\circ}$ south, from where a two-sector distribution was seen until almost at the south pole, when the spacecraft possibly moved south of the heliospheric current sheet (HCS) [Smith et al., 2001]. The dipolar pattern was again detectable at the spacecraft from immediately after maximum southern latitudes until mid-northern latitudes, when Ulysses moved north of the HCS. The sector structure was next detectable when Ulysses returned to midlatitudes. Although plasma observations during the solar maximum fast latitude scan revealed a highly-complex mixture of flows [McComas et al., 2002], magnetic field observations during the same period showed a surprisingly simple picture, similar to that expected for a simple dipole field with axes close to the solar equator [Jones and Balogh, 2003]. We restrict the results presented here to the data returned from the latter part of the first orbit to the end of 2002.

\section{Data Analysis}

\subsection{Ballistic Mapping of Data to the Solar Wind Source Surface}

[5] The HMF polarity distributions were derived from hourly-averaged Ulysses magnetometer [Balogh et al., 1992] and solar wind data [Bame et al., 1992]. The polarity at the spacecraft was estimated by comparing the instantaneous Parker spiral direction, calculated using solar wind velocity measurements, with the actual field azimuth angle. For each data point, ballistic mapping was used to estimate the source Carrington longitude of the parcel of plasma represented by that datum. This made use of the coordinates of the observation's sub-Ulysses point on the Sun, the solar wind velocity at the spacecraft, the spacecraft's heliocentric distance, and the rotation period of the Carrington reference 


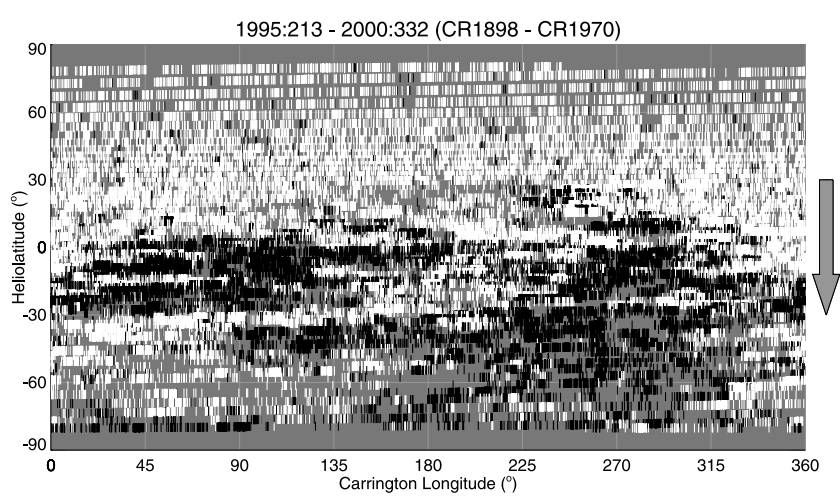

2000:333 - 2001:286 (CR1970 - CR1981)

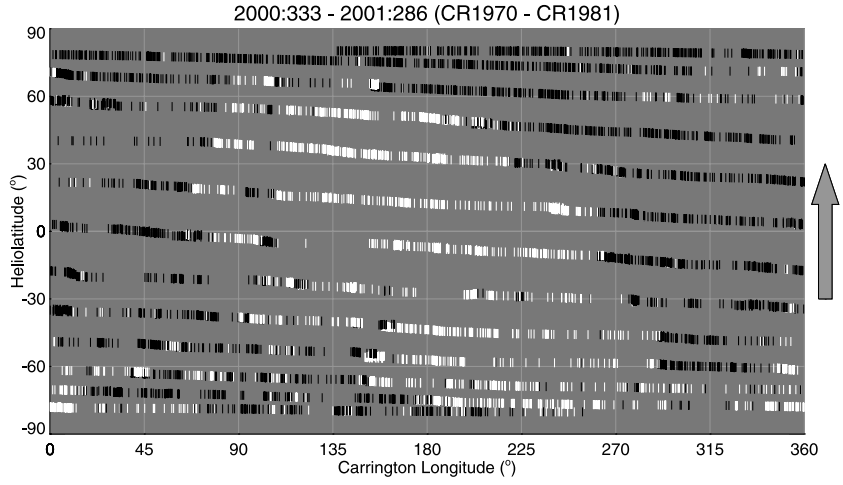

2001:287 - 2002:365 (CR1981 - CR1998)

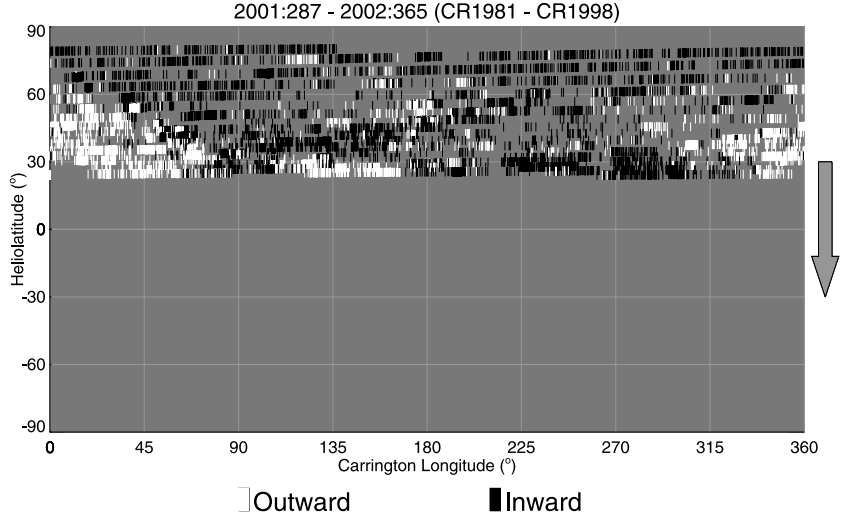

Figure 1. Hourly-averaged HMF polarity observations at Ulysses during 1995:213-2002:365, mapped back to the solar source surface. The Carrington Rotation range is given for each panel. Arrows show the direction of change in Ulysses's heliolatitude. The polarities are shown as white (outwards, or positive) or black (inwards, or negative).

frame ( $=25.38$ days in the inertial frame). The solar wind was assumed to have remained at a constant heliolatitude between the source surface and spacecraft. The Carrington rotation during which the plasma left the Sun was also recorded, to simplify comparison with results from other sources. Some uncertainties are known to be associated with some of the assumptions made in ballistic mapping [e.g., Neugebauer et al., 1998], but the results are believed to be sufficiently accurate for an overview of the polarity distribution.

\subsection{Determination of the Dipole Tilt}

[6] Analysis of the sector structure at Ulysses can provide a means of estimating the dipole tilt over time. A significant simplifying assumption has to be made i.e., that the field is purely dipolar, with a flat, warp-free HCS. This disagrees with the actual HCS topology [e.g., see the review of HCS work by Smith, 2001], but, as shown below, this assumption provides remarkably consistent results.

[7] When not over the solar equator, the footpoint of the solar wind plasma encountered by Ulysses describes a small circle on the solar wind source surface (Figure 2). The principle used to estimate the dipole tilt was that, using the ratio of outward (positive) to inward (negative) fields at the spacecraft during each solar rotation, the dipole tilt with respect to the solar rotational equator can be estimated. Only when the dipole axis passes through the equatorial plane will a ratio of $1: 1$ be seen in the two magnetic polarities at all heliolatitudes.

[8] The mapped-back data described above were separated according to the Carrington rotation (CR) in which the plasma left the Sun. For each CR, the mean heliolatitude of Ulysses was noted. As large variations in the solar wind velocity, which were common during the latest fast latitude scan, could lead to very large changes in the spatial distribution of the source longitudes, the mapped data were then reduced further. Times of uncertain polarities, defined by the field being $60^{\circ}-120^{\circ}$ from the Parker spiral, were ignored. Most high-latitude magnetic field polarity inversions, which are clearly unrelated to HCS crossings [Balogh et al., 1999], were removed from the data by this step. The source surface was divided into $5^{\circ}$ bins in Carrington longitude for each $\mathrm{CR}$, and the dominant polarity in each bin determined. As well as removing the biasing caused by velocity variations, this re-sampling removed the effect of

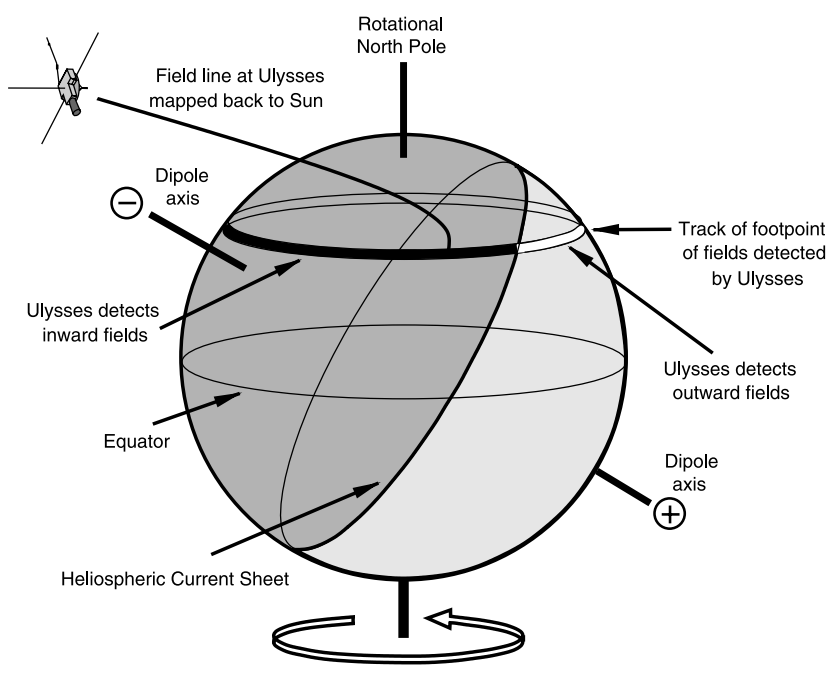

Figure 2. Sketch illustrating the technique used to estimate the dipole tilt angle. The sphere represents the solar wind source surface, at $\sim 2.5$ solar radii. The source of solar wind encountered by Ulysses describes a small circle on this sphere of approximately constant heliolatitude. When the footpoint of this material is in the positive hemisphere of the dipole, outward fields are detected; the opposite is true if the footpoint is in the negative hemisphere. The ratio of positive to negative polarities provides an estimate of the tilt angle. In this example, Ulysses samples wind predominantly originating in the negative hemisphere. 
the overlap in source longitudes, which is inherent in data mapped back from Ulysses's near-polar orbit [Neugebauer et al., 2002 and references therein]. Empty bins were filled using interpolation. For each rotation, a $360^{\circ}$ distribution of source surface polarities was thus obtained.

[9] Dipole tilts derived from near-equator data were extremely sensitive to polarity ratio variations, as a 1:1 ratio is expected there, by definition. The data within a neararbitrary heliolatitude range of $\pm 10^{\circ}$ were thus ignored. Periods when the spacecraft did not cross the HCS during the course of a solar rotation did not, by definition, provide unique values of dipole tilt angle. Limits on the tilt angle could however be derived for these periods; these were purely dependent on the spacecraft's heliolatitude. Such periods defined most of Ulysses's first polar orbit. Although transient structures produced times of "false" polarity reversals, it was decided not to attempt to remove these features from the data set, as overall, they were expected to contribute approximately the same proportion of extra outward and inward polarity periods to the data.

\section{Results}

\subsection{Rate of Reversal of the Dipole Component}

[10] Figure 3 shows the dipole tilt angle, defined as the angle between the solar north rotational pole and the positive pole of the magnetic dipole, estimated using the above technique. Although there is a scatter in the results during some periods, the underlying trend in the angle is clear. The dipole axis changed from being near-parallel to the rotational axis to near-orthogonal in $\sim 2.5$ years, and since early 2001 has continued to evolve towards a nearantiparallel alignment. Reversal (i.e., when the dipole axis crossed the equator) probably took place between early 2000 and early 2001.

[11] The scatter in tilt angles is significant, particularly during 1999-early 2000, and mid-late 2002. The anomalous result for CR1975 may have been due to Ulysses's proximity to the equator, lying just outside the ignored $\pm 10^{\circ}$ heliolatitude band. We also note that the sector structure changed significantly at certain times, as seen in Figure 1. The period from the equator to $\sim 30^{\circ} \mathrm{S}(\mathrm{CR} 1930-1951)$ was defined by a four-sector structure, suggesting a strong quadrupolar component at that time, and raising questions about the applicability of the dipole assumptions to that period. Sanderson et al. [2003] do report a mixed dipolar/ quadrupolar field at this time. At the very end of 2002, the polarities again began to show a four-sector structure, probably reflecting the existence of warps in the HCS.

[12] The assumption of a flat HCS is probably a major cause of the scatter. The HCS location can easily be influenced by the position of active regions [Forsyth et al., 1997], and a realignment of fields following major relaxation of coronal fields following flares may give rise to abrupt changes in the HCS's topology. Large changes in the HCS may occur in less than a solar rotation, giving the false impression that Ulysses spent a long time within a certain polarity, when actually it would have spent a much shorter time had the HCS location remained constant. Transient events could have had a strong influence during certain periods, introducing "false" polarity measurements. The large number of interplanetary counterparts of coronal

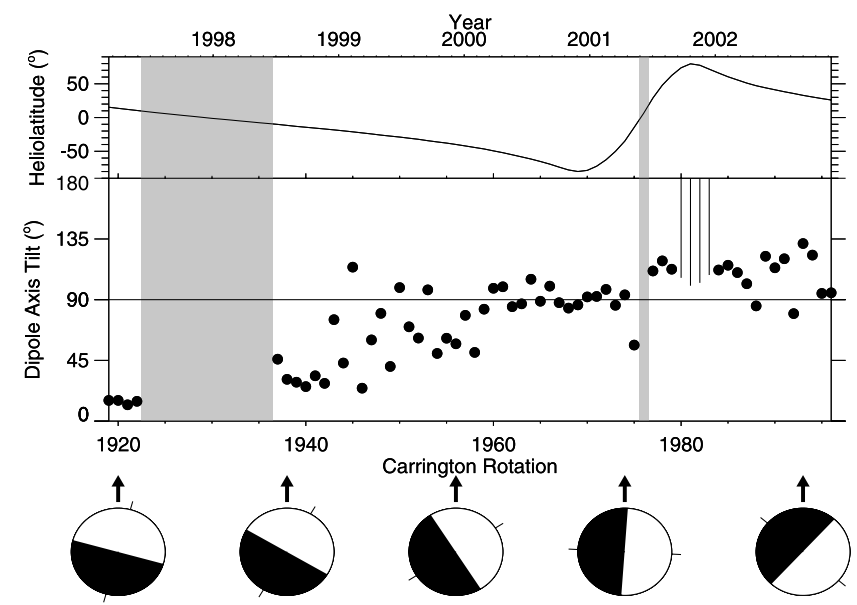

Figure 3. Variation in the dipole axis angle derived from Ulysses data. Top panel: heliospheric latitude of Ulysses. Lower panel: dipole tilt, defined as the angle between the dipole axis and the solar equator (zero when positive pole coincident with north rotational pole). Gaps occur when Ulysses is within $10^{\circ}$ of the solar equator. When Ulysses was in unipolar fields, only a range of possible tilt angles could be derived; these are represented by vertical lines. Representations of the reversing dipole are shown at bottom.

mass ejections (19 during the fast latitude scan alone [Forsyth et al., 2003]), probably had a significant influence on the data.

\subsection{Motion of the Dipole Component Axis in the Carrington Reference Frame}

[13] The fitting of a dipole to the sector structure at Ulysses revealed clear motion of the dipole in the Carrington reference frame during several periods, including that encompassing the fast latitude scan. This can be seen in the slant of the positive sector in the middle panel of Figure 1. This longitude drift corresponds to a $\sim 26.5-26.8$ day rotation period in the sidereal frame. Dipole rotation rates of this order have been suggested in previous works (e.g., Svalgaard and Wilcox [1975], and in Ulysses data by Balogh et al. [1993]). The persistence of this drift to very high heliolatitudes is reported here for the first time. Whilst noting the proposed explanation for the drift in terms of the eruption and transport of flux in active regions [e.g., Wang et al., 1988, and references therein], we draw attention to a potentially significant fact: These rates agree well with the rotation rates inferred from helioseismological studies for the region of the solar interior where the magnetic dynamo is suspected of operating. The rotational frequency of $\sim 432-437 \mathrm{nHz}$, equivalent to the longitude drift, correlates with that inferred to exist in the outer radiative zone, within the tachocline [Thompson et al., 1996]. Mechanisms proposed to account for the current sheet rotation [e.g., Fisk and Schwadron, 2001] generally involve processes occurring near the solar surface, rather than in the interior. Neugebauer et al. [2000] reported on a synodic periodicity of 27.03 days in solar wind speed and the radial component of the HMF at several spacecraft over three solar cycles, consistent with the magnetic dipole returning to the same 
longitude during each reversal. That rate is faster than that reported here.

\section{Discussion and Conclusions}

[14] Using Ulysses data and positional information alone, constraints have been obtained on the tilt of the solar magnetic dipole. Analysis of the magnetic field reversal using remote observations was carried out by Wang et al. [2002] and Sanderson et al. [2003]. The work of the former team indicates that reversal did not occur simultaneously at both poles: The streamer brightness peaks occurred over the North and South poles in February and October 2000 , respectively. Source surface fields from photospheric measurements indicated that reversal occurred at the North and South Poles by February and July 2000, respectively (CR1959 and 1964). Ulysses's observations near $80^{\circ} \mathrm{S}$ in November 2000 (CR 1969) suggested that reversal had not occurred by that time [Smith et al., 2001]. The South pole was however tipped away from the Earth during late 2000, significantly affecting the source surface model. Wang and co-workers showed that multiplying the strength of the negative polarity by 3 over the whole of the "hidden" southern polar cap during that time produced a source surface field consistent with the Ulysses observations of negative polarities at the highest southern latitudes during CR1969.

[15] The results presented here suggest that polarity reversal, i.e. when the dipole axis crossed the solar equator, occurred around CR1960-CR1970, and cannot be tied down to a period shorter than several months. This agrees well with the indicators of polarity reversal used by Wang et al. [2002]. Sanderson et al. [2003] inferred that reversal occurred in late 2000, which again is in good agreement with our inferences. However, they infer that the positive magnetic pole was almost at the southern rotational pole at the end of 2001. Our results indicate that the dipole tilt was still very high $\left(\sim 110^{\circ}\right)$ at that time. Although the results presented here indicate a gradual rotation of the dipole, we note that observed occasional sudden, $\sim 180^{\circ}$ shifts in longitude of the sector structure [e.g., Jones and Balogh, 2003] are not consistent with a simple rotation of the dipole component.

[16] By studying source surface models that include dipolar as well as higher-order terms, Wang et al. [2002] state that the reversal cannot be described as a simple rotation of the dipole. The results presented here show that when the HMF observations are interpreted in purely dipolar terms, the rotation of the dipole component at least can be followed through solar maximum. It could be argued that the similarity of the solar maximum fast latitude scan data to a simple dipole with its axis near the equatorial plane could be a sampling effect, caused by changes in the heliospheric field occurring in step with Ulysses's motion. The source surface models described by Sanderson et al. [2003] show that the dipole term can dominate even at maximum solar activity. It appears that the Ulysses observations reflect such a situation, particularly around the fast latitude scan period.
[17] The continued return of data from Ulysses will provide invaluable and unique information on the dipole's behaviour over the new solar activity cycle. A valuable future study would involve the fitting of dipole models to the Ulysses data when combined with similar information obtained at low latitudes.

[18] Acknowledgments. Ulysses research at Imperial College London is supported by the UK Particle Physics and Astronomy Research Council. D. J. McComas is thanked for the use of SWOOPS data. We are grateful to Y.-M. Wang and N. R. Sheeley for useful comments.

\section{References}

Balogh, A., et al., The magnetic field investigation on the Ulysses mission: Instrumentation and preliminary scientific results, Astron. Astrophys. Suppl. Ser., 92, 221-236, 1992.

Balogh, A., G. Erdös, R. J. Forsyth, and E. J. Smith, The evolution of the interplanetary sector structure in 1992, Geophys. Res. Lett., 20(21), 2331, 1993.

Balogh, A., R. J. Forsyth, E. A. Lucek, T. S. Horbury, and E. J. Smith, Heliospheric magnetic field polarity inversions at high heliographic latitudes, Geophys. Res. Lett., 26(6), 631-634, 1999.

Bame, S. J., et al., Ulysses solar-wind plasma experiment, Astron. Astrophys. Suppl. Ser., 92, 237-265, 1992.

Fisk, L. A., and N. A. Schwadron, The behavior of the open magnetic flux of the Sun, Astrophys. J., 560, 425-438, 2001.

Forsyth, R. J., A. Balogh, E. J. Smith, and J. T. Gosling, Ulysses observations of the northward extension of the heliospheric current sheet, Geophys. Res. Lett., 24, 3101, 1997.

Forsyth, R. J., A. Rees, D. B. Reisenfeld, and S. T. Lepri, ICME observations during the Ulysses fast latitude scan, Proceeding of Solar Wind X, M. Jelli et al., eds., AIP conference proceedings, 679, 715, 2003

Jones, G. H., and A. Balogh, The global heliospheric magnetic field polarity distribution as seen at Ulysses, Ann. Geophys., 21(6), 1377, 2003.

McComas, D. J., H. A. Elliott, J. T. Gosling, D. B. Reisenfeld, R. M. Skoug, B. E. Goldstein, M. Neugebauer, and A. Balogh, Ulysses' second fast-latitude scan: Complexity near solar maximum and the reformation of polar coronal holes, Geophys. Res. Lett., 29(9), 10.1029/ 2001GL014164, 2002.

Neugebauer, M., et al., The spatial structures of the solar wind and comparisons with solar data and models, J. Geophys. Res., 103(A7), 14,58714,599, 1998.

Neugebauer, M., E. J. Smith, A. Ruzmaikin, J. Feynman, and A. H. Vaughan, The solar magnetic field and the solar wind: Existence of preferred longitudes, J. Geophys. Res., 105(A2), 2315-2324, 2000.

Neugebauer, M., P. C. Liewer, E. J. Smith, R. M. Skoug, and T. H. Zurbuchen, Sources of the solar wind at solar activity maximum, J. Geophys. Res., 107(A12), 1488, 10.1029/2001JA000306, 2002.

Sanderson, T. R., T. Appourchaux, J. T. Hoeksema, and K. L. Harvey, Observations of the Sun's magnetic field during the recent solar maximum, J. Geophys. Res., 108(A1), 1035, doi:10.1029/2002JA009388, 2003.

Smith, E. J., The heliospheric current sheet, J. Geophys. Res., 106(A8), 15819-15831, 2001.

Smith, E. J., A. Balogh, R. J. Forsyth, and D. J. McComas, Ulysses in the south polar cap at solar maximum: Heliospheric magnetic field, Geophys. Res. Lett., 28(22), 4159-4162, 2001.

Svalgaard, L., and J. M. Wilcox, Long term evolution of solar sector structure, Solar Phys., 41, 461-475, 1975.

Thompson, M. J., et al., Differential Rotation and Dynamics of the Solar Interior, Science, 272, 1300-1305, 1996.

Wang, Y.-M., N. R. Sheeley Jr., A. G. Nash, and L. R. Shampine, The quasi-rigid rotation of coronal magnetic fields, Astrophys. J., 327, 427$450,1988$.

Wang, Y. M., N. R. Sheeley, and M. D. Andrews, Polarity reversal of the solar magnetic field during cycle 23, J. Geophys. Res., 107(A12), 1465, 10.1029/2002JA009463, 2002.

A. Balogh and G. H. Jones, Space and Atmospheric Physics Group, The Blackett Laboratory, Imperial College London, London, SW7 2BW, UK. (g.h.jones@imperial.ac.uk)

E. J. Smith, Jet Propulsion Laboratory, 4800 Oak Grove Drive, Pasadena, CA 91109, USA. 